\title{
Tratamento cirúrgico com sucesso de tromboembolismo pulmonar maciço
}

Danton R. da Rocha LOURES*, Iseu Affonso da COSTA*, Yukio SUZUKI*, Luiz LOPES*, Américo F. MARTINS* , José C. MULASKI*, Gastão Pereira da CUNHA**, Cláudio Pereira da CUNHA**

\section{RBCCV $44205-81$}

LOURES, D. R. R.; COSTA, I. A.; SUZUKI, Y.; LOPES, L.; MARTINS, A. F.; MULASKI, J. C.; CUNHA, G. P.; CUNHA, C. P. - Tratamento cirúrgico com sucesso de tromboembolismo pulmonar maciço. Rev. Bras. Cir. Cardiovasc., 4(1): 90-93, 1989.

RESUMO: É relatado caso de tromboembolismo pulmonar maciço em paciente de 63 anos de idade com grave comprometimento hemodinâmico e cujo diagnóstico foi confirmado por ecocardiografia bidimensional; submetido a embolectomia puimonar, realizada 52 horas após a internação, recebeu alta hospitalar no $43^{\circ}$. dia de pós-operatório.

DESCRITORES: tromboembolismo pulmonar, cirurgia.

\section{RELATO DO CASO}

J. L. T., masculino, 63 anos de idade, cor negra, foi internado no dia 24/04/88 com queixa de "dor no peito" e dispnéia aos pequenos esforços, dispnéia paroxística noturna e ortopnéia há 10 anos. Dois dias antes da internação, sentiu súbita dor precordial, tipo pontada, ventilatória dependente. Ao exame físico, apresentava pressão arterial de $110 / 90 \mathrm{mmHg}$, pulso de $92 \mathrm{p} / \mathrm{m}$, freqüência respiratória de $20 \mathrm{p} / \mathrm{m}$ e temperatura de $37,3^{\circ}$ centígrados; choque da ponta no $5^{\circ}$. espaço intercostal com bulhas cardiacas rítmicas, hiperfonéticas e atrito pericárdico; pulmōes livres, com boa expansibilidade; presença de úlcera varicosa e edema de membros inferiores. Os exames complementares revelaram, ECG: infarto de parede inferior e ântero-septal, bloqueio de ramo direito e isquemia subepicárdica de parede anterior; gasometria sagüínea: $\mathrm{ph}=7,36 ; \mathrm{pO}^{2}=67,9 ; \mathrm{pcO}^{2}=$ 32,3 ; bicarbonato $=18,0 ; \mathrm{BB}=42,2 ; \mathrm{BE}=5,8$; as enzimas não evidenciaram infartc do miocárdio recente e a radiografia do tórax mostrava lesão subpleural direita compativel com tromboembolismo. Foram aventadas as hipóteses diagnósticas de angina pós-infarto, pericardite com infarto do miocárdio e tromboembolismo pulmonar. A ecocardiograíia realizada em 27/04 mostrou imagem bastante móvel deslocando-se entre átrio direito e ventrículo direito de aspecto atípico, que sugeria trombo móvel (Figura A1/A2). No dia seguinte, a dor persistia com aumento da freqüência respiratória e com a presença de 3: bulha cardiaca. Às 18:00 horas, ao ser encaminhado ao centro cirúrgico para retirada de trombos das cavidades direitas, ocorreu parada cardiaca durante a esternotomia. O coração foi reanimiado e instalada a circulação extracorpórea. O lado direito do coração estava tenso e distendido. Não foram observados trombos no átrio e/ou ventrículo direito, mas a arteriotomia do tronco pulmonar demonstrou trombo "a cavaleiro" localizado a nivel da bifurcação associado a outros trombos menores em diversos ramos e subdivisōes da árvore arterial pulmonar. (Figura B). A manobra cirúrgica consistiu na retirada cautelosa com auxilio de uma pinça de Winter de trombos. No período de pós-operatório, permaneceu 9 dias em unidade de terapia intensiva (UTI), para cuidados especiais com assistência respiratória controlada, débito cardíaco e do equilibrio metabólico. Após alta da UTI, foi realizada uma cintilografia pulmonar de

\footnotetext{
Trabalho realizado no Departamento de Cirurgia e no Departamento de Clínica Médica da Universidade Federal do Paraná. Curitiba, PR. Brasil. Recebido para publicação em 20 de abril de 1989.

- Do Departamento de Cirurgia da Universidade Federal do Paraná

** Do Departamento de Clínica Médica da Universidade Federal do Paraná.

Endereço para separatas: Danton da Rocha Loures. Rua Augusto Stellfeld, 2088. Bigorrilho. 80430 Curitiba, PR, Brasil.
} 
LOURES, D. R. R.; COSTA, I. A.; SUZUKI, Y.; LOPES, L.; MARTINS, A. F.; MULASKI, J. C.; CUNHA, G. P.; CUNHA, C. P. - Tratamento cirúrgico com sucesso de tromboembolismo pulmonar maciço. Rev. Bras. Cir. Cardiovasc., 4(1): 90-93, 1989.

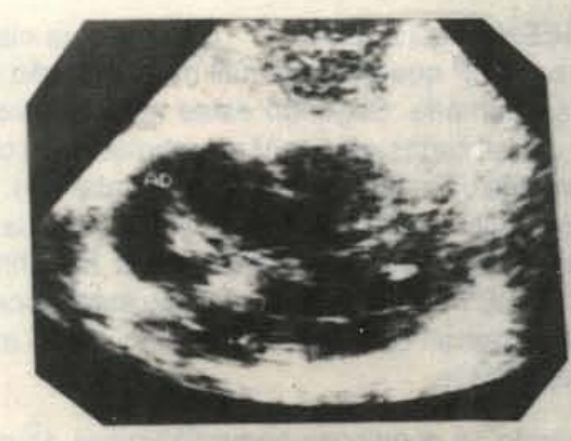

A1

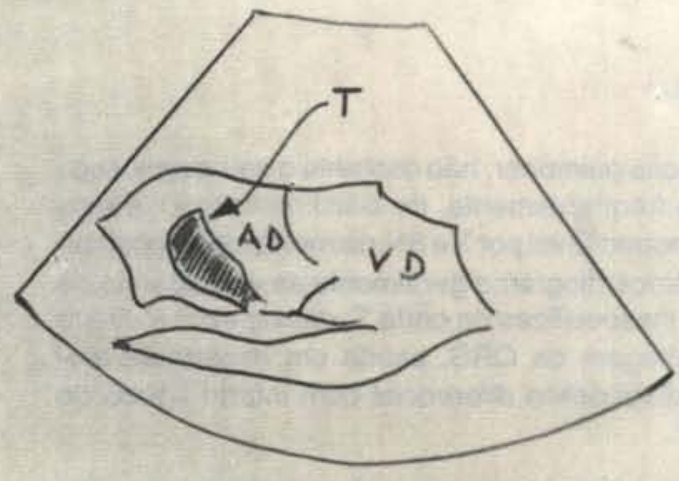

A2

Fig. A1 e A2 - Ecocardiograma bidimensional por via subcostal mostrando trombo (T) no interior do átrio direito (AD). Na imagem dinâmica, durante o exame, observa-se movimentação errática de massas no $A D$ que se dirigiam também até o ventrículo direito (VD).

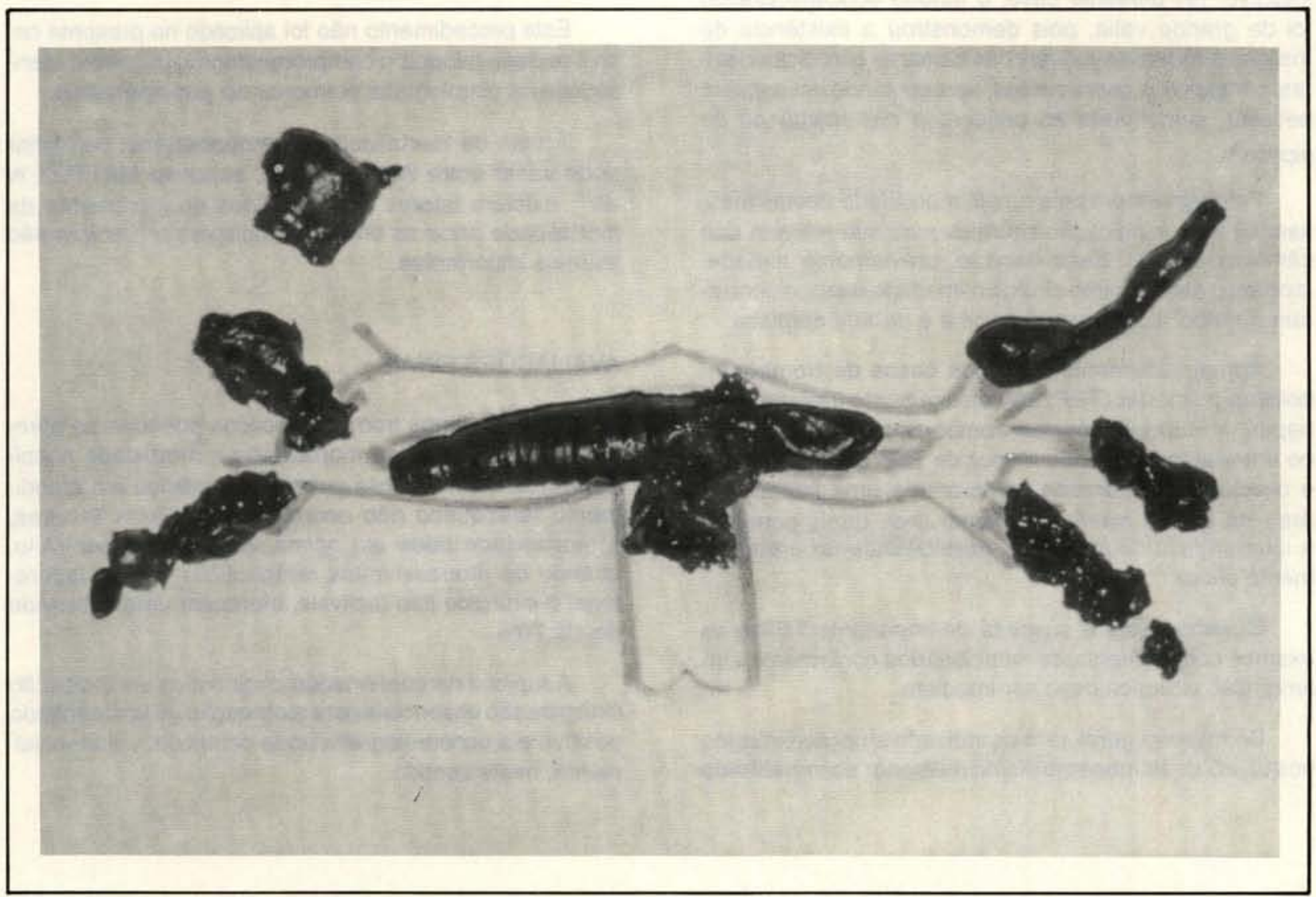

Fig. B - Trombos retirados durante a cirurgia e dispostos sobre um desenho do tronco e artérias pulmonares de maneira semelhante ao encontrado no ato cirúrgico. 
LOURES, D. R. R.; COSTA, I. A.; SUZUKI, Y.; LOPES, L.; MARTINS, A. F.; MULASKI, J. C.; CUNHA, G. P.; CUNHA, C. P.

- Tratamento cirúrgico com sucesso de tromboembolismo pulmonar maciço. Rev. Bras. Cir. Cardiovasc., 4(1): 90-93, 1989.

controle, que revelou acentuada diminuição de perfusão à esquerda e do lobo médio direito. Recebeu alta hospitalar no 43 : dia de pós-operatório com a recomendação do uso de cumarínico.

\section{DISCUSSÃO}

A embolia pulmonar, não osbtante o seu diagnóstico seja, ainda freqüentemente, de difícil realização, é estimado ser responsável por 3 a $5 \%$ da mortalidade hospitalar $^{3}$. O eletrocardiograma geralmente se acompanha de alteraçōes inespecíficas da onda $\mathrm{T}$, desvio axial à direita e baixa voltagem de QRS, sendo um importante elemento no diagnóstico diferencial com infarto agudo do miocárdio ${ }^{2}$.

A cintolografia pulmonar, a tomografia computadorizada e a ecocardiografia podem ser realizadas prestando auxílio diagnóstico significativo. A ecocardiografia bidimensional tem-se mostrado de valor na detecção de trombos e massas intracardiacas ${ }^{6}$, sendo indicada rotineiramente na pesquisa de fontes emboligenas. Em alguns casos, têm sido diagnosticados êmbolos no tronco pulmonar de maneira mais precisa, após o evento da ecocardiografia transesofágica, assim obtendo-se um diagnóstico de certeza através de um meio rápido e nãoinvasivo. No presente caso, o estudo ecocardiográfico foi de grande valia, pois demonstrou a existência de massas ecogênitas móveis nas câmaras cardiacas direitas, compativeis com trombos, apresentando um aspecto peculiar, semelhante ao observado nas máquinas de pipoca ${ }^{6}$.

Pelo tamanho e pela ampla mobilidade destas massas, foi feita a indicação cirúrgica para sua retirada das câmaras direitas. Essa decisāo, previamente tomada, facilitou $o$ atendimento cirúrgico imediato quando ocorreram a embolia pulmonar maciça e a parada cardíaca.

Aproximadamente $90 \%$ dos casos de tromboembolismo pulmonar (TEP) são efetivamente tratados com heparina e/ou substâncias tromboembolóticas ${ }^{7}$. Existe, no entretanto, um grupo menor de TEP onde o número e o volume de trombos compromete uma importante área da árvore arterial pulmonar, nos quais somente a interferência cirúrgica tem possibilidade de um tratamento eficaz ${ }^{10}$.

Quando existe a suspeita de importante TEP e os exames complementares mencionados confirmam, a intervenção cirúrgica deve ser imediata.

De maneira geral, tem-se indicado atuação cirúrgica nos casos de tromboembolismo pulmonar acompanhado de choque cardiogênico, hipoxemia, acidose metabólica, pressão média da artéria pulmonar maior que 20-30 $\mathrm{mmHg}$, pressão venosa central elevada e oclusão de tronco pulmonar igual ou superior a $50 \%$.

GREENFIELD \& ZOCCO $^{4}$ criaram uma classificação para o TEP que pode ser útil na orientação da conduta a ser tomada. Segundo estes autores, nos casos de TEP classificados em IV e V, a embolectomia cirúrgica tem oferecido os melhores resultados (Tabela 1). Segundo ROBINSON et alii $^{8}$, a primeira embolectomia pulmonar realizada com sucesso deve-se a Kirschner, em 1924. COOLEY' realizou com sucesso este procedimento a "céu aberto" com o auxílo da circulação extracorpórea, em 1961.

Quando as condiçōes hemodinâmicas do paciente estão desfavoráveis no momento da indicação cirúrgica, a possibilidade de haver uma parada cardíaca durante a toracotomia existe e a abordagem das femorais pode oferecer um excelente auxilio terapêutico, permitindo o manuseio cardíaco e a instalação da circulação extracorpórea total.

Durante a embolectomia pulmonar a "céu aberto", recomenda-se massagem pulmonar centrípeta visando "ordenhar" os trombos remanescentes da periferia para o hilo ${ }^{9}$.

Este procedimento não foi aplicado no presente caso e poderia minorar o comprometimento pulmonar identificado na cintolografia pulmonar no pós-operatório.

A taxa de mortalidade da embolectomia pulmonar pode variar entre $24 \%$ a $93 \%$ e, segundo MATTOX et alii ${ }^{5}$, existem fatores bem definidos de incremento da mortalidade onde as embolias múltiplas e maciças săo os mais importantes.

\section{AVALIAÇÕES FINAIS}

Os fenômenos tromboembólicos pulmonares apresentam altas taxas de mortalidade e morbidade hospitalar. Quando a embolia pulmonar é maciça e o atendimento terapêutico năo ocorre nas primeiras 6 horas, a mortalidade pode ser acima de $85 \%$. Ao contrário, quando os procedimentos terapêuticos clínico, laboratorial e cirúrgico são factíveis, oferecem uma sobrevida de até $70 \%$.

A rapidez na confirmação diagnóstica e a indicação cirúrgica são essenciais para a obtenção de um resultado positivo e a ecocardiografia pode contribuir, substancialmente, neste sentido. 
LOURES, D. R. R.; COSTA, I. A.; SUZUKI, Y.; LOPES, L.; MARTINS, A. F.; MULASKI, J. C.; CUNHA, G. P.; CUNHA, C. P. - Tratamento cirúrgico com sucesso de tromboembolismo pulmonar maciço. Rev. Bras. Cir. Cardiovasc., 4(1): 90-93, 1989.

TABELA 1

CLASSIFICAÇĀO DO TROMBOEMBOLISMO PULMONAR

\begin{tabular}{clccl}
\hline CLASSE & SINTOMASISINIAIS & GASES & AP OCLUSÃO (\%) & HEMODINAMICA \\
\hline I & Ausentes & Normal & Menor que $20 \%$ & Normal \\
II & Ansiedade - Hiperventilação & $\mathrm{pO}^{2} 80 \mathrm{mmHg}$ & 20 a $30 \%$ & Taquicardia \\
III & Dispnéia - Colapso & $\mathrm{pO}^{2} 65 \mathrm{mmHg}$ & 30 a $50 \%$ & $\begin{array}{l}\text { PVC elevada } \\
\text { PA pulm. } 20 \mathrm{mmHg}\end{array}$ \\
IV & Dispnéia - Choque & $\mathrm{pO}^{2} 50 \mathrm{mmHg}$ & maior que $50 \%$ & PVC elevada \\
& & $\mathrm{pCO}^{2} 30 \mathrm{mmHg}$ pulm. $25 \mathrm{mmHg}$ & PVC elevada \\
V & Dispnéia & & & PA pulm. $40 \mathrm{mmHg}$ \\
Débito cardíaco baixo sem \\
choque
\end{tabular}

GREENFIELD \& $\mathrm{ZOCCO}^{4}$.

$\mathrm{AP}=$ artéria pulmonar; $\mathrm{pO}^{2}=$ pressão de oxigênio; $\mathrm{pCO}^{2}=$ pressão de gás carbónico; $\mathrm{PVC}=$ pressão venosa central; Pa pulm $=$ pressăo de artéria pulmonar.

RBCCV 44205-81

LOURES, D. R. R.; COSTA, I. A.; SUZUKI, Y.; LOPES, L.; MARTINS, A. F.; MULASKI, J. C.; CUNHA, G. P.; CUNHA, C. P. - Massive pulmonary thromboembolism successful surgical treatment. Rev. Bras. Cir. Cardiovasc., 4(1): 90-93, 1989.

ABASTRACT: The authors report a case of massive pulmonary thromboembolism in a 63 year old patient with a severe impairment of hemodynamic condition. The clinical diagnostic was confirmed by $2 \mathrm{D}$ echo and the patient underwent successful pulmonary embolectomy, 52 hours after admission and was discharged on the 34rd postoperative day.

DESCRIPTORS: pulmonary thromboembolism, surgery.

\section{REFERÊNCIAS BIBLIOGRÁFICAS}

1 COOLEY, D. A. - Techniques in cardiac surgery. New York, W. B. Saunders Company, $1984 . \quad$ p. 131.

2 DI CARLO Jr., L. A.; SCHILLER, N.; HERFKENS, R. L.; BRUNDAGE, B. H.; LIPTON, M. J. - Noninvasive detection of proximal pulmonary artery thrombosis by two-dimensional echocardiography computerized tomography. Am. Heart J., 104: 879-881, 1982.

3 GRASSFOR, D. M.; ALFOR, W. C.; BURRUS, G. R. Pulmonary embolectomy. Ann. Thorac. Surg., 32: 28-32, 1981.

4 GREENFIELD, L. J. \& ZOCCO, J. J. - Intraluminal management of acute massive pulmonary thromboembolism. J. Thorac. Cardiovasc. Surg., 77: 402-410, 1979.

5 MATTOX, K. L.; GELDTMAN, R. W.; BEALL, A. C.; DeBAKEY, M. E. - Pulmonary embolectomy for acute massive pulmonary embolism. Ann. Surg., 195: 726-730, 1982.
6 NASSER, F. N. \& GIULIANI, E. R. - Cardiac masses in clinical two dimensional echocardiography. Chicago, Year Book Medical Publishers Inc., 1983. p. 187-206.

7 PARASKOS, J. A.; ADELSTEIN. S. J.; SMITH, R. E.; RICKMAN, R. D.; GROSSMAN, W.; DEXTER, L.; DALEN, J.E. - Late prognosis of acute pulmonary embolism. N. Engl. J. Med., 289: 55-58, 1973.

8 ROBINSON, R. J.; FEHRENBACHER, J.; BROWN, J. W.; MADURA, J. A.; KING, H. - Emergency pulmonary embolectomy: the treatment for massive pulmonary embolus. Ann. Thorac. Surg., 42: 52-55, 1986.

9 SABISTON Jr., D. C. - Trendelemburg's classic work on the operative treatment of pulmonary embolism. Ann. Thorac. Surg., 35: 570-574, 1983.

10 TSCHIRKOV, A.; DRAUSE, E.; ELERT, O.; SATTER, P. - Surgical management of massive pulmonary embolism. J. Thorac. Cardiovasc. Surg., 75: 730-733, 1978. 